\title{
Three-dimensional mapping and comparative analysis of the distal human corpus cavernosum and the inflatable penile prosthesis
}

\author{
Tariq S Hakky ${ }^{1}$, Daniel Ferguson ${ }^{2}$, Philippe E Spiess ${ }^{1}$, Paul Bradley ${ }^{1}$, Tom F Lue ${ }^{3}$ and Rafael E Carrion ${ }^{1}$
}

The intricate anatomy of the corpus cavernosum in both the flaccid and tumescent state has not been fully elucidated. We report our experience using a three-dimensional (3D) scanner to reconstruct cadaveric casts and compare them with 3D images of two prototypes of penile prosthesis. Two different models of the Titan Coloplast inflatable penile prosthesis were analyzed using a 3D scanner. The first was the standard model and the second was a newer model with a rounder silicone tip. Two cadaveric phalluses were harvested using Smooth-Cast 300Q polyurethane molding. The molds were excised and scanned along side the penile prosthesis. 3D scans were completed and analyzed using Leios Mesh software, and GOM Inspect software. The 3D scans demonstrated the mean human corporal radii $2 \mathrm{~mm}$ from the distal tip to be $36.51 \mathrm{~mm}(36.01-37.0 \mathrm{~mm})$, which is an obtuse angle. The standard Titan penile prosthesis spherical radius at the same level was $202.52 \mathrm{~mm}$, while the new silicone tip prosthesis had a radius of $139.33 \mathrm{~mm}$. 3D mapping further demonstrated the trajectory of the cavernosa appeared curvilinear and the distal ends appeared blunt. The use of cadaveric cavernosal molds in combination with the 3D scanner allowed us to accurately image the corpus cavernosum for the first time. Our findings suggest that anatomically accurate corporal tips appear to be relatively blunt and that the new Titan silicone tip penile prosthesis more closely resembles the human corporal tip.

Asian Journal of Andrology (2013) 15, 567-570; doi:10.1038/aja.2012.172; published online 1 April 2013

Keywords: corpus cavernosum; penile prosthesis; three-dimensional analysis

\section{INTRODUCTION}

Since the placement of the first penile implant in 1936 by Nikolaj Bogaraz, the penile prosthesis has evolved considerably. ${ }^{1,2}$ There has been much improvement in the design, mechanical reliability and ease of use over the last 35 years. Penile prosthesis surgery is a widely accepted treatment for men with erectile dysfunction refractory to pharmacologic therapy. It is associated with satisfaction rates of greater than $90 \% .{ }^{3}$ With all of the advancements made to the penile prosthesis, the intricate anatomy of the distal corpora cavernosa in relation to the distal end of the penile prosthesis has not been fully elucidated.

Careful dissection of the human corpora cavernosa demonstrates a paired erectile system that originates as separate structures under each wing of the ischium. ${ }^{4,5}$ As they extend forward under the pubic symphysis side-by-side, they share a septum medially, which is perforated allowing for communication of the sinuses distally. ${ }^{4-6}$ Both gross and microscopic analysis have been performed on the human corpora, yet we lack incite on the spatial and anatomic features of distal tips of the human corpus cavernosum.

Advancements in medical imaging technology have greatly improved our understanding of the corpus cavernosum. Improvements in computed tomography (CT) and magnetic resonance imaging (MRI), offer superior definition of soft-tissue planes and definition of penile tissue. ${ }^{7}$ Nevertheless, scans are not performed with subjects in the erect state and deformation artifacts cause a lack of fine detail limiting CT and MRI images. Rapid advances in computer technology along with three-dimensional (3D) computer-aided digitizers have improved our understanding of the human body. 3D scanners offer unprecedented accuracy of up to $50 \mu \mathrm{m}$. 3D digitizers has been used to assist health care providers in fields such as craniofacial surgery during facial reconstruction. ${ }^{8}$ By using this new technology, we hope to gain a better anatomic understanding of the distal human corpora cavernosa and its relationship with the distal tip of the inflatable penile prosthesis.

Over the past century, increased comprehension of the penile anatomy has been gained through scientific study. Advancements have guided surgical practice, and industry products. Both improved technique, and a firm grasp of the male human anatomy has yielded to fewer penile prosthesis complications with improved functional and aesthetic results. We believe that every urologist should have a firm understanding of the anatomy of the native corpora cavernosa in comparison to that of the penile prosthesis, which resides within it.

\section{MATERIALS AND METHODS}

Two cadaveric pelvises of men aged 71 and 81 years were obtained. Exclusion criterion included any cadaver, which had any prior penile prosthetic surgery, cavernosography, or any cavernosal manipulation or plication. A Foley catheter was placed into each urethra to aid with

${ }^{1}$ Department of Urology, University of South Florida, 2 Tampa General Circle, Tampa, FL 33602, USA; ${ }^{2}$ 3D3Solutions, Burnaby, BC V5J 5M8, Canada and ${ }^{3}$ Department of Urology, University of Southern California San Francisco, 400 Parnassus Ave, San Francisco, CA 94143, USA Correspondence: Dr RE Carrion (rcarrion@health.usf.edu)

Received: 19 November 2012; Revised: 16 December 2012; Accepted: 20 January 2013; Published online: 1 April 2013 
dissection and identification of anatomy. Smooth-Cast 300 Q polyurethane molding was then injected into the corpora using a 16 gauge needle with $60 \mathrm{cc}$ syringe seen in Figure 1. Once a full tumescence was achieved using the molding cast, the penises were allowed to sit for $5 \mathrm{~min}$ while the mold hardened. Once this was complete, the penises were carefully dissected from the pelvises taking care not to injure the corpora. After successful extraction of both molds, they were then soaked in sodium hydroxide to remove all remaining human tissue for $12 \mathrm{~h}$. Once this was complete the cadaveric molds were then scanned using a 3D scanner. All of the four corpora cavernosal tips were then analyzed.

Two different model Titan Coloplast (Minneapolis, MN, USA) inflatable penile prostheses were obtained for this study. Both prosthetics were $18 \mathrm{~cm}$ in length and had never been used in situ. The first prosthetic was the current standard Titan penile prosthesis, and the latter was the newer silicone tip Titan penile prosthesis. Both of the prosthesis were separately scanned and analyzed.

The FlexScan HDI Advance R2 3D Scanner from 3D3 Solutions (Burnaby, BC, Canada) was utilized to scan all targets. Prior to scanning each object in the field, the scanner was calibrated using $9 \times 12$ calibration grid of $10 \mathrm{~mm}$ to establish a field of view of $140 \mathrm{~mm}$ for all shapes. The scanner was used to capture a series of 8 scans around the perimeter of the target item. The individual 3D surfaces were aligned and merged to a continuous 3D surface using Leios software from EG Solutions. A final global alignment algorithm in Leios was used to ensure the $3 \mathrm{D}$ volume error was less than $0.1 \mathrm{~mm}$. Further analysis was performed by individually analyzing the four corpora cavernosa by creating $2 \mathrm{D}$ cross-sections, and fitting curves to the scanned surface planes using GOM Inspect software from GOM mbH (Braunschweig, Germany). The final reporting was generated using GOM Inspect. Distal radial curvature analysis was performed on all targets using Rhino 3D from McNeel and Associates (Seattle, WA, USA). The color curve mapping was performed on all shapes using Pro/Engineer WF5 from PTC (Needham, MA, USA).

\section{RESULTS}

The length of the human Corpora 1 was measured to be $13.3 \mathrm{~cm}$ and $19.7 \mathrm{~cm}$ for Corpora 2. The specimen Corpora 1 had Peyronie's disease

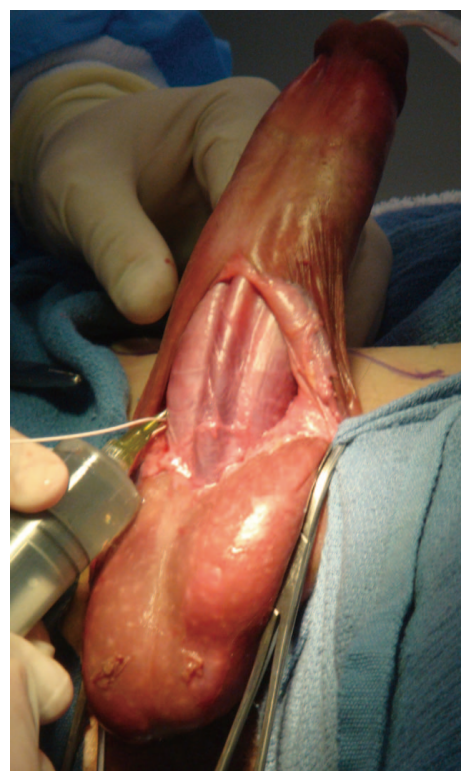

Figure 1 Injection of smooth cast with 16-gauge needle while harvesting the corpus cavernosum.



b

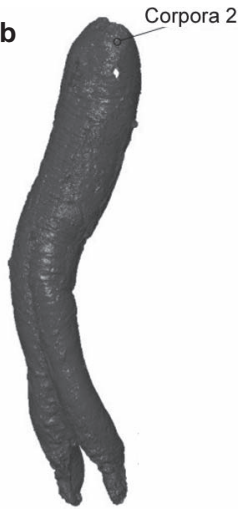

Figure 2 Three-dimensional reconstructions of both human corpus cavernosum.

limited to the mid shaft level (Figure 2a) with a $78.3^{\circ}$ dorsal right curve. Corpora 2 had a curvature of $14.8^{\circ}$ dorsal and left (Figure $2 \mathbf{b}$ ). The maximum diameter of the corpora was measured to be $2.0 \mathrm{~cm}$ and $2.1 \mathrm{~cm}$ respectively. Both of the maximum diameter values for the corpora were reached distally. The volumes of the erect human corpus cavernosum measured in our study were $862.2 \mathrm{~cm}^{2}$ and $930.0 \mathrm{~cm}^{2}$.

Analysis of an interpolated 3D curve was generated from the digitized scans of the erect corpora cavernosa and penile prosthesis cylinders. In order to compare the radius of our target objects, we analyzed the differences in radii of extrapolated cross-sectional fitted circles. The circles were analyzed $2 \mathrm{~mm}$ from the distal tip of each corpora cavernosa and the tip of each prosthetic. These measurements were obtained in radii where smaller values signify more rounded tips. The four human corpora cavernosa were measured and the mean spherical radii at $2 \mathrm{~mm}$ from the distal tip was $36.51 \mathrm{~mm}(36.01 \mathrm{~mm}-37.0 \mathrm{~mm})$. The standard Titan penile prosthesis spherical radii at the same $2 \mathrm{~mm}$ distance from the tip was $202.52 \mathrm{~mm}$; however, the new silicone tip prosthesis had radii of $139.33 \mathrm{~mm}$ at the same depth. The new silicone tip Titan penile prosthesis more closely resembles the human corporal radii. The differences in spherical radii and slope $2 \mathrm{~mm}$ from the distal tip of each object are seen in Figures 3 and 4. The new silicone tip penile prosthesis better conforms to the tip of the rounder human corpora cavernosa. It was observed that the trajectory of the cavernosa appeared curvilinear and the distal ends appeared less acute.

Figure 5a is a color map of the surface averaging the spherical radii of all of the human corpora. The new silicone tipped penile prosthesis (Figure $\mathbf{5 b}$ ) is seen in the middle and the standard penile prosthesis (Figure 5c) on the far right. The color map was created by analyzing

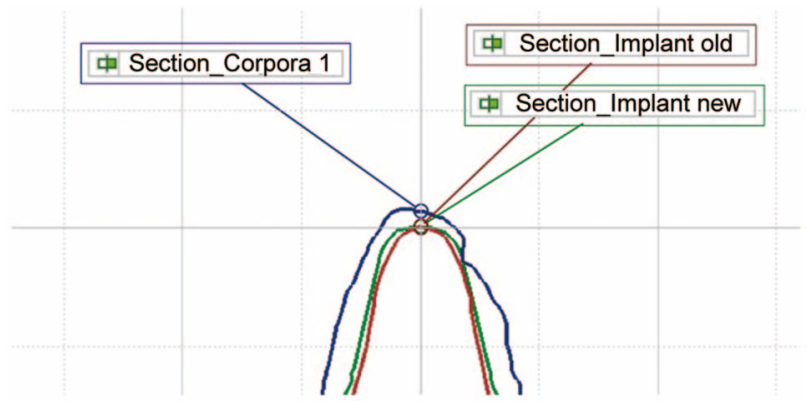

Figure 3 Two-dimensional cross-section image of the standard Titan penile prosthesis. The new silicone tip prosthesis superimposed over a two-dimensional image of the distal corpora cavernosa. This highlights the difference in angle curvature at the tip. One can see the new silicone tip has a blunt curve. 


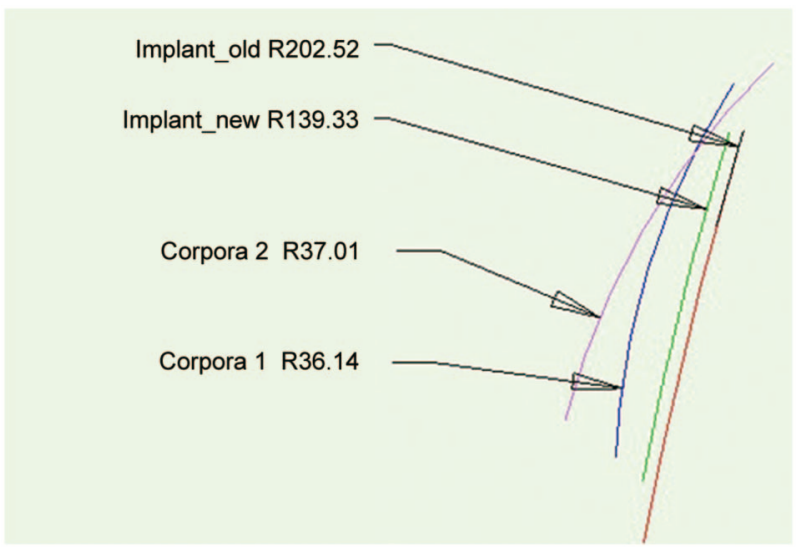

Figure 4 The radii and slopes of the corpora and implants listed from the 2D cross-sectional analysis by fitted circles $2 \mathrm{~mm}$ from the distal tips of each target object. One can appreciate the differences in slopes at $2 \mathrm{~mm}$ from the distal tip of each of the target objects. 2D, two-dimensional.

the different radii of extrapolated cross-sectional fitted circles at $1 \mathrm{~mm}$ intervals from the distal tip of each object. The surface radii were then averaged and compared to the two penile prosthesis cylinders. This color map demonstrates data points in spherical radii denoted by color. The averaged color mapping surface of the tips of the human corpora (Figure 5a) yielded low spherical radii or a relatively flat surface that is denoted in the color blue. The new silicone tip penile prosthesis (Figure $\mathbf{5 b}$ ) has a larger blue tip than the standard penile prosthesis (Figure 5c) indicating a closer match in radii to the human corpora. The larger spherical radii are signified as a topographical rise or a sharp slope. These data are seen in the colors green, yellow and red (denoted in increasing order or slope values). The layers of blue, green and red on the map (Figure 5) demonstrate different radii values, which both the human corpora and the penile prosthesis exhibit in a similar tiered fashion. The color mapping of the human corpora has a smooth transition zone from blue to green to yellow and finally red without overlapping colors owing to its gentle slope as the corpora expands. However, both of the Titan penile prostheses appear to have a red band in the color map highlighting a difference in shape and spherical radii between them and the corpora. This red band rise signifies a sharp topographical rise or sharp slope when compared to the human corpora's relatively gentle slope. Additionally, the new silicone tip prosthesis (Figure $\mathbf{5 b}$ ) has a larger blue-green band in the transition zone when compared to the standard prosthesis (Figure 5c). This larger band translates to a gentler curve or a lower slope while the prosthesis is transitioning sizes. This affirms that the human corpus cavernosum have a relatively gentle curved tip with a mild slope in the transition zone. We also demonstrate the new silicone tipped penile prosthesis more closely resembles the human corpora when compared with the standard prosthesis.

Limitations of our study include our sample size of four corpora cavernosa, and the advanced age of the corpora. We also did not analyze corpora that previously been dilated or that had a penile prosthetic previously within it. Another limiting factor is computer error, which was calculated at less than $1 \mathrm{~mm}$. An additional limitation is that one of the patients had Peyronie's disease; however, this patient's disease was limited to the mid shaft and the distal tips of the corpora cavernosa were not affected. More importantly, the difference in spherical radii between the tips of both cadavers was $1 \mathrm{~mm}$ regardless if the patient had Peyronie's disease.

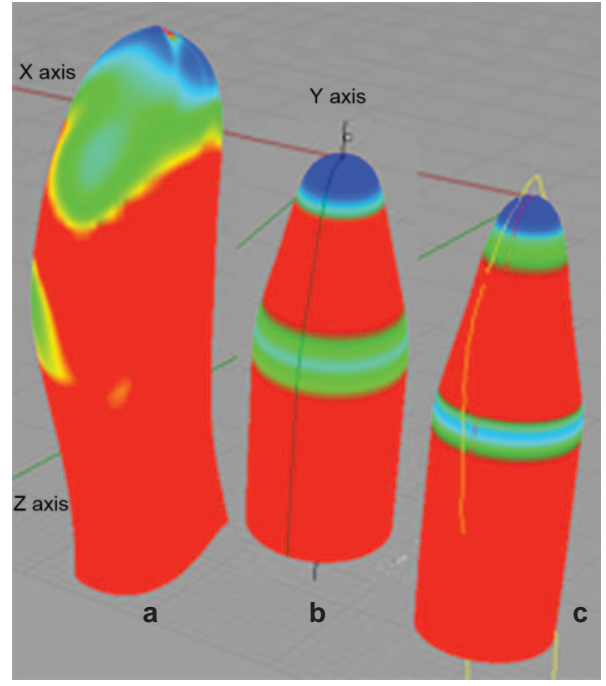

Figure 5 (a) 3D mapping of the human corpora denoted in spherical radii. (b) 3D mapping of the new silicone tipped Titan penile implant. (c) 3D mapping of the standard tip Titan implant. 3D, three-dimensional.

\section{CONCLUSIONS}

Cavernosography has given urologists access to some of the earliest images of the erect corpus cavernosum. Through the use of 3D mapping and computer analysis, we have been able to examine the architecture of the erect distal corpus cavernosum and the distal tips of the penile prosthesis cylinders.

Today, the penile prosthesis remains the surgical cornerstone in the treatment of erectile dysfunction. For the first time, we were able to use 3D mapping and scanning of both erect human corpus cavernosum and the inflatable penile prosthesis to give us some insight into the interaction of this device and the tips of human corpora. Surprisingly, the distal human corporal tips appear rounded with low spherical radii values when measured $2 \mathrm{~mm}$ from the tip. 3D mapping demonstrated curved tips with a smooth transition zones throughout the distal erect corpora. The new silicone tip Titan penile prosthesis appears to better conform to the tip of the human corpora. This possibly translates into less sheering forces on the human corpora, and a more natural fit and feel.

Further investigation is necessary to analyze the proximal and distal corpus cavernosum along with its relationship to the penile prosthesis cylinders. This will propel the evolution of the penile prosthesis to have a better fit and feel along with an improved understanding of the mechanical forces it sustains. Additionally, auxiliary tests are required to explore the relationship of the corpora and the cylinder before and after dilation and prosthetic placement. As manufactures of the penile prosthetics strive to improve their products, further work is needed to analyze the intricate relationship between the human corpora and the inflatable penile prosthesis.

\section{AUTHOR CONTRIBUTIONS}

TSH harvested corpora, wrote the manuscript, helped organize project. DF performed three dimensional analysis. PB harvested corpora, wrote the manuscript and edited the image. PES harvested corpora and wrote the manuscript. TFL wrote the manuscript. REC harvested corpora, wrote the manuscript and organized the project.

\section{COMPETING FINANCIAL INTERESTS}

The authors declare no competing financial interests. 
neg

Distal human corpus cavernosum

570

TS Hakky et al

ACKNOWLEDGMENTS

Funding for the cadavers and prosthesis was by Coloplast Corporation.

1

Wahle GR, Mulcahy JJ. Ventral penile approach in unitary component penile prosthesis placement. J Ural 1993; 149: 537-8.

2 Le TF, Mason R, Risen R, Guiliano F, Khoury S et al. Sexual Medincine: Sexual Dysfunction in Men and Women. Paris: Health Publications Limited; 2004. p475-7.

3 Deveci S, Martin D, Parker M, Mulhall JP. Penile length alterations following penile prosthesis surgery. Eur Urol 2007; 51: 1128-31.

4
5 Le TF, Hon S. Physiology of penile erection and pathophysiology of erectile dysfunction. In: Win AJ, Kavoussi LR, Novick AC, Partoin AW, Peters CA, editors. Campbell Urology. 10th ed. Philadelphia, PA: Elsevier; 2011. p691.

6 Hsu GL, Brock G, Martinez-Pineiro L, won Hoyden B, Le TF et al. Anatomy and strength of the tunic abuginea: Its relevance to penile prosthesis extrusion. $J$ Ural 1994; 151: 1205-8.

7 McPhail EF, Nehru A, Bruner BC, Kawashima A, King BF et al. MRI and its role in the evaluation and surgical decision making in patients with challenging IPP presentations: descriptions of MRI findings and algorhythm for patient management. BJU Int 2012; 109: 1848-52.

8 Ozsoy U, Demirel BM, Yildirim FB, Tosun O, Sarikcioglu L. Method selection in craniofacial measurements: advantages and disadvantages of $3 \mathrm{D}$ digitization method. J Cranio-Maxillofacial Surg 2009; 37: 285-90.

Asian Journal of Andrology 